\title{
Le Sud et le monde nouveau
}

Yu Jian, Frank Muyard

\section{Citer ce document / Cite this document :}

Jian Yu, Muyard Frank, Tianya. Le Sud et le monde nouveau. In: Perspectives chinoises, n45, 1998. pp. 18-24;

doi : https://doi.org/10.3406/perch.1998.2248

https://www.persee.fr/doc/perch_1021-9013_1998_num_45_1_2248

Fichier pdf généré le 24/04/2018 


\section{Le}

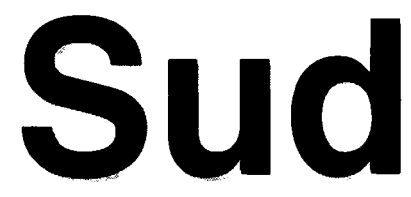

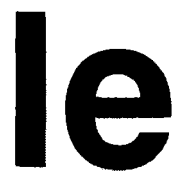

E texte de Yu Jian présenté ici fuł publié en février 1997 à l'occasion d'un débat sur la Chine ef la modernisation dans la revue chinoise Tianya ${ }^{(1)}$. II est écrit par l'un des poètes contemporains chinois les plus en vue. Né en 1954 à Kunming, où il vit toujours, Yu Jian acquit une grande notoriété en 1994 quand la pièce "L'autre rive » (Bi'an), montée par Mou Sen à I'Institut du Cinéma de Pékin, ébranla le monde intellectuel de la capitale. Basée sur un de ses textes, "Discussion linguistique à propos de l'autre rive " ${ }^{(2)}$, la pièce, écrite en collaboration avec Gao Xingiian, mettait en cause la pensée du " meilleur des mondes » au cœur des utopies et idéologies politiques, notamment communiste, qui ont traversé la Chine du XXe siècle. Sa critique radicale de l'idée de progrès, mais aussi de tout "paradis», ainsi que son approche linguistique, marquèrent aux yeux de la critique littéraire une césure dans la création contemporaine. On abattait tout d'un coup les assises du modernisme au profit d'une pensée "post-moderne » saluée comme le retour de « la raison » à la réalité ${ }^{[3]}$. Couronné par la revue littéraire taiwanaise Lianhe bao (Unitas) pour un de ses recueils, Yu Jian vit un autre de ses poèmes, "Dossier zéro" (Ling dang'an) transposé au théâtre par Mou Sen en 1995. Présentée au festival de Créteil et au Festival international des Amériques de Montréal, cette pièce relatait dans un double monologue le drame personnel des familles de « mauvaise » origine sociale lors de la Révolution culturelle, et l'écrasement des individualités par le système de dossier idéologique mis en place par le pouvoir communiste pour contrôler la vie de la population chinoise.

On retrouve dans le texte traduit ici tant sa critique de la Révolution culturelle que celle de l'idéologie moderne, notamment dans son application à la Chine. Contrairement aux articles d'un Liu Xiaobo ${ }^{(4)}$ qui tentent de promouvoir la pensée critique de la modernité des Lumières, Yu Jian, en appelle, lui, par le biais d'une référence à Heidegger, à un retour a l'idéal traditionnel chinois de l'union de l'homme et du ciel. En ceci, il pourrait se placer dans la ligne de pensée néo-conservatrice qui tend à dominer les cercles intellectuels chinois ces dernières années ${ }^{(5)}$. Il fait, par ailleurs, une critique acerbe de certains aspects de la société chinoise. Il épingle notamment le rapport excessivement utilita- riste des Chinois au monde animal et végétal, la déprofessionalisation générale de la société après 1949, et le désintérêt des élites pour les problèmes de la pollution et de la destruction de l'environnement.

Mais la position de Yu Jian est aussi la position marginale d'un homme du Sud qui défend sa culture locale. Les deux thèmes principaux de l'article, volontairement mis en opposition, sont ainsi ceux de l'identité culturelle de la Chine du Sud et de la poursuite du «nouveau " monde. L'union d'un fort régionalisme, d'une assimilation du «moderne » au «nouveau » et à ses conséquences néfastes sur l'environnement, et d'un rappel de l'éthique "naturaliste» classique, prend alors bien les couleurs d'un post-modernisme sinisé qui, s'appuyant sur l'« échec » du monde moderne occidental et la fin des "grands récits", met en avant une " sinité » ou des « valeurs asiatiques » à emploi local. L'image du Sud défendue ici pourra sembler très éloignée de la culture moderne d'autres "Sud" de la Chine, comme Shanghai ou Hong Kong. Elle accompagne, en fait, la relecture généreuse de la tradition chinoise par $Y_{u}$ Jian qui, si elle en laissera plusieurs sceptiques, est représentative des courants culturalistes du moment.

De fait, l'article de Yu Jian met en lumière l'impasse pratique et intellectuelle dans laquelle la Chine, et luimême, se trouvent plongés: d'un côté, la poursuite aveugle du profit et la perte de la culture et des savoirs traditionnels, de l'autre, la recherche angoissée de racines identitaires, le repli traditionaliste et le maintien d'une critique sociale et culturelle à un niveau éthique toujours pré-politique.

Frank Muyard

1. Yu Jian, "Nanfang yu xin shijie ", Tianya (Frontiers), Haikou (Hainan), 1997, n², pp. 9.13.

2. "Dui bi'an yici hanyu cixing taolun ", Jinri xianfeng (Avantgarde actuelle), 1995, n I (Pékin). Autres œuvres importantes, "Soixante poèmes de Yu Jian " (Shi liushi shou), Yunnan renmin chubanshe, 1988, "Nommer un corbeau " (Dui yizhi wuya de mingmingl, Guoji wenhua chubanshe, 1993.

3. Voir le documentaire de Jiang Yue, "L'autre rive " (Bi'an), 1995, qui relate le processus de création de la pièce, et ses répercussions tant sur son public que sur ses acteurs.

4. Voir les deux articles de Liu Xiaobo in Perspectives chinoises $n^{\circ}$ 40, mars/avril 1997, pp. 29-37.

5. Voir Chen Yan, «Les courants d'idées en Chine», Perspectives chinoises $n^{\circ} 36$, juillet/août 1996, pp. 6.12. 


\title{
monde nouveau
}

\author{
YU JIAN \\ TIANYA, FÉVRIER 1997, PP. 9-13 \\ TRAIUIT DU CHINOIS PAR FRANK MUYARD
}

\section{A propos de l'époque et de la tradition}

"Suivre la marche de l’époque » est le mot d'ordre dont les intellectuels chinois du XXe siècle furent le plus fermement convaincus. Il a marqué l'évolution tant de l'avant-garde politique du début du siècle que de l'avant-garde artistique de la fin de siècle. Dans la langue chinoise du $\mathrm{XXe}$ siècle, les mots «neuf» et «vieux" perdirent ainsi très vite leur sens neutre. Neuf signifia l'avant-garde, la révolution, le positif, l'ascension, le bon. Vieux prit le sens d'arriéré, de réactionnaire, de cc qui va être anćanti, de ce qui cst à l'article de la mort. Le tournant de gauche des intellectuels des années 1930. l'extension de la campagne anti-droitiste de 1957, la «Révolution culturelle » de 1966, la mode du départ à l'étranger des années 1980, la Nouvelle Vague artistique, tout comme le "saut dans les affaires » et la «post-critique » des années 1990 résultent tous de cette tendance à « suivre la marche de l'époque ». I a peur d’être halayé par l'époque, l'empressement à entrer dans le courant du moment sont devenus un modele de vie, une parole dautorité. Ainsi, dans la littérature contemporaine. partout la critique de la vie et des personnages à la Oblomov est prise pour vérité établie. Ce pays a non seulement mis au ban l'ancien à travers des mouvements politiques excessivement simplificateurs. mais il a encore profondément planté la haine de tout ce qui est vieux dans la langue quotidienne du peuple. C'est pour- quoi en Chine il y a des vieux mais peu d'Anciens, il y a de vieux meubles mais peu d'esprit de conservation, il y a de l'histoire mais peu de tradition. La tradition c'est toujours ce que l'on rejette avec mépris. Si les gens font bien parfois un retour au passé, c'est seulement quand ce passé peut servir le nouveau. Ne peuvent-ils donc pas s'arrêter un peu et réfléchir sur cette drôle de chose qu'on appelle «époque » et dont ils suivent la marche? Quelle est-elle? Qui estelle? Est-ce une chose concrète, faite de sang, qui a une vie? Pourquoi a-t-cllc un si fort pouvoir d'attraction sur nous? Au point qu'elle remplacerait notre réflexion? Qu'elle nous ferait abandonner à la légère notre village natal, l'amour des jours anciens, les collines de poiriers en fleur, la vieille maison de grand-mère, les vieilles boutiques, et le dialecte ou la langue maternelle de notre bout de pays? Pourquoi est-ce que nous avons aussi peur de nous faire appeler des «ploucs", des « vieux »? Si l'on doit personnifier l'époque. sera-t-elle « je »? «tu»? «il »? De toute évidence elle ne peut être représentée par une seule personne, car si elle a du poids c'est que c'est un phénomène de masse. Elle ne peut être que «nous» ou «eux». En fait. le mot époque sert souvent. en chinois, à désigner «nous". Suivre la marche du temps signifie suivre " notre » marche. Être délaissé par l'époque. c'est être abandonné dans un " je » sans " nous".

Combien de gens cette drôle de chose qu'on appelle époque a-t-elle déjà détruits! Beaucoup lui imputent ainsi leur incapacité à écrire, que ce soit la campagne anti-droitiste de 1957, la « Révolution culturelle », ou l'économie de marché... pourtant, il n'y a jamais eu dans l'histoire ce qu'on pourrait appeler un Age d'or de l'écriture. Les gens qui ne peuvent pas écrire, quelle que soit l'époque, n'arrivent pas à écrire, mais ceux qui peuvent écrire écrivent toute leur vie. Je ne crois donc pas qu'il y ait de soi-disant époques où l'on ne peut pas écrire, et tous ceux qui donnent ce prétexte pour leur abandon de l'écriture, ont, selon moi, une autre but dans la vie.

Dans ce dernier siècle de révolution ininterrompue, d'avant-gardisme et de réforme du vieux monde, un grand nombre de gens, en suivant la marche de l'époque, se sont, spirituellement ou techniquement, transformés en amateurs. Il manque maintenant d'employés fidèlement professionnels, il manque d'éléments conservateurs, de vieux irréductibles. Alors que le professionnalisme signifie la norme, le dilettantisme c'est «tout peut convenir ». S il y a une norme, on l'échange tout de suite contre argent comptant. Le monde post-moderne du profit réel a. dans ce pays, une base sociale idéologique naturelle.

Dans les années 1950, quelques intellectuels versés dans la culture chinoise recommandèrent que le nouveau pays se dirige vers la professionnalisation. Ce fut rejeté. Le résultat en fut l' " amateurisation» générale de la Chine. Chaque per- 
sonne alla s'occuper des "grandes affaires ». chaque personne crut au soi-disant «le monde est à nous». Le coeur de l'époque se trouva alors sur la place publique et non dans les ateliers. Au lieu de "à chacun sa tâche ", la règle fut "au premier appel, tout le monde accourt $»$. Ceci est la marque d'une société dilettante. Une autre de ses conséquences est la méfiance générale envers le professionnalisme, qui fut si forte que lors des périodes extrêmes, des mesures de répression furent prises contre tout savoir spécialisé, toute «expertise ». La critique de la voie "bourgeoise" du professionnalisme conduisit finalement à la perte générale des normes de qualité de vie, alors que la norme idéologique, elle, uniformisa tout à un point jamais vu. Or, si la norme idéologique peut être changée alternativement par le pouvoir, la perte des normes générales de qualité de vie ne peut en aucun cas être annulée en un court laps de temps, car leur reconstruction nécessite une accumulation progressive de la qualité de vie. Aujourd'hui, la norme idéologique fondamentale a changé. C'est, disons-le, l'objectif d'une vie conforme au bonheur des gens. Mais on n'en hérite pas moins dans la vie quotidienne des résultats désastreux de la perte des normes professionnelles.

La prétendue conscience malheureuse des intellectuels chinois est ramenée ainsi en fait toujours aux arguties de «où va-t-on?" ou de "la situation actuelle et nos tâches ». Pendant qu'ils font de grands discours sur «la perte de l'âme ", ces intellectuels restent pourtant aveugles devant la pollution urbaine, la question des canalisations de gaz des petits quartiers, ou encore la démolition en série des maisons avec une cour (siheyuan) pour le seul profit des promoteurs immobiliers. Cette conscience malheureuse est de fait complice de ses propres malheurs. J'estime que si, en dehors de notre spécialité, on veut à ses moments libres se pen-

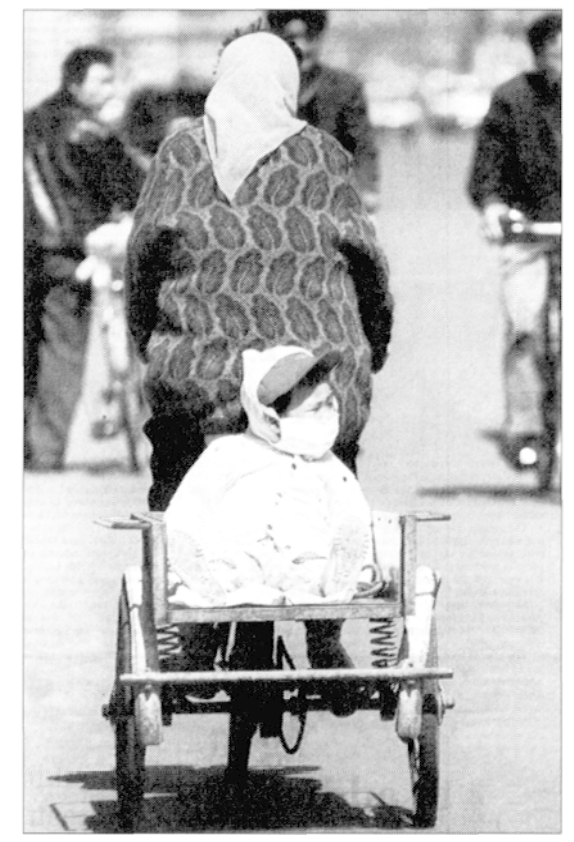

La pollution:

un vrai problème de société

cher sur les malheurs de la société, il vaudrait mieux, au lieu de discuter de la «profondeur spirituelle» et autres questions oiseuses de ce style, simplement débattre des problèmes concrets de la planification urbaine et de la pollution de l'environnement. Mais les intellectuels ne daignent même pas y accorder un regard. Dans ce pays, si vous discutez de la justesse de la ligne idéologique, il vous sera facile de briller et de gagner les faveurs du public. Mais si vous discutez du problème de la pollution du fleuve Panlong à Kunming, la majorité des gens préféreront boire cette eau sale que de donner le moindre avis sur la question. Ah, si seulement on avait pu dans les années 1950 discuter de la question démographique soulevée par Ma Yinchu (1) et de celle de la transformation de la vieille ville de Pékin avancée par Liang Sicheng (2) avec autant de ferveur que de la pièce "La destitution de Hairui » (3) ou encore du débat d’il y a deux ans sur la perte de l'esprit humaniste! Mais ce n'est même pas concevable!

\section{A propos du Sud et de la langue}

Dans l'histoire chinoise, le Sud a toujours représenté une culture marginale. La riche variété des conditions géographiques du Sud a déterminé la multiplicité des formes d'existence de ses habitants, favorisant dans leur rapport au monde le développement d'une capacité d'imagination créative. La culture dominante chinoise a principalement grandi dans le Nord et la plaine centrale, là où la nature est relativement aride et pauvre, et les conditions de vie très difficiles. Un monde gris sans nuances s'adapte beaucoup mieux aux guerres de grande échelle et à l'unification impériale. Mais il rend souvent perplexe un homme du Sud qui aime ardemment la vie et qui vient d'un monde à la topographie complexe, parsemé de hautes montagnes et de grands fleuves, de forêts et de sources, de rocs et de ravins, au ciel éternellement bleu, aux mets délicieux et aux populations autochtones simples et candides.

$\mathrm{Au}$ XXe siècle, le symbole linguistique de la nouvelle unification de la société chinoise fut, dans les années 1950, l'extension du mandarin à travers la formation d'un grand nombre de poètes à la création dans cette langue. La dernière manifestation de cette empreinte fut visible dans le couronnement pour "profondeur de sentiments » d'écrits possédant « un niveau spirituel » et qui n'était en fait que la reconnaissance d'une communauté de sensibilité avec les critiques et les poètes de la «langue du Nord» (qui n'est pas le dialecte de Pékin de Lao She).

Mais le Sud s'est toujours obstiné dans sa différence et c'est pourquoi il est souvent un casse-tête pour le centre. Partant de Shanghai, remontant le cours du Yangzi, dépassant le bassin du Sichuan et jusquaux confins chinois du Yunnan, le mandarin est vu partout comme le mode d'expression des haut-parleurs et des éditoriaux officiels. Héritant de la tradition de Qu Yuan, au Sud, c'est le dialecte qui est la langue nourricière de la poésie.

Le Sud désigne un monde en per- 
pétuel bouillonnement, tandis que le Nord est le «jardin des plaisirs». On peut le voir au niveau de la langue locale. Tous les Chinois peuvent comprendre le mandarin fondé sur la langue du nord, mais seuls les Shanghaïens, les Sichuanais, les Yunnanais... peuvent comprendre leur dialecte local.

Le président de la Biennale de Venise a dit une phrase au sujet du monde de langue anglaise qui s'applique également au monde sinophone : "Dans chaque pays, le Sud n'est pas du tout un lieu géographique, et en général, présente encore moins de conditions favorables au développement économique. Il symbolise plutôt un lieu de création artistique. Là, chaque individu va manifester sa capacité d'imagination dans une forme artisanale et cachée pour résister à là culture dominante. En ce sens, le Sud représente un espace artistique modèle, un espace où l'imagination individuelle résiste à l'environnement externe. C'est ainsi que le Sud a donné naissance à de nombreux poètes qui ne savaient s'exprimer clairement que dans la langue de leur région natale et qui ne se sentaient à l'aise que dans la vie quotidienne.

Dans le Nord de la Chine. le mandarin, étant considéré comme le symbole du monde nouveau, a pénétré depuis longtemps toutes les régions dialectales. Pékin, en tant que centre politique et culturel de la Chine, suscite évidemment le respect des Chinois et du monde entier. Mais la vie traditionnelle typique de l'humanité confucéenne de la Chine du Nord, telle qu'elle pouvait être exprimée dans l'emploi du dialecte pékinois chez Lao She, a déjà graduellement disparu à Pékin. Un Pékin qui perd ses maisons à cour et ses murailles bâties sous les Ming et les Qing, un Pékin de langue mandarine, est au niveau de la vie quotidienne un échantillon type du monde nouveau, à la fois le point de convergence et le baromètre de la politique chinoise, l'exposition cul- turelle universelle et le quai de l'import-export, le modèle. Mais la situation du Sud n'est pas la même. Dans le Sud des littératures de langue yue (cantonaise), wu (Jiangnan) et autres — jadis dénombrées par Hu Shi - le mandarin est un peu l'anglais de la langue chinoise, un simple outil de communication entre des cultures différentes. Le Sud de la Chine, dernier bastion de la vie quotidienne traditionnelle chinoise, est difficilement unifiable par le mandarin. Pourtant, on peut se faire du souci pour sa capacité de résistance. Parce qu'il n'y avait aucune idéologie pratique capable de défendre la société chinoise traditionnelle, après 1966, le développement de la culture chinoise est devenu, en effet, souvent vide de sens. En revanche, le monde nouveau passa très vite du statut de slogan des intellectuels radicaux minoritaires du début du siècle au rang d'idéologie concrète dominante. Aujourd'hui, les gens savent ainsi généralement copier toutes les manières d'entrer dans le monde nouveau, mais ils ne savent pas comment préserver la tradition qui est en eux. Aujourd'hui, ce qui pénètre l'univers intérieur des enfants comme des vieillards, ce n'est plus la pensée close, et moribonde, de la société confucéenne. C'est l'appel et la mise en ouvre d'une vie moderne aux changements quotidiens qui est devenue la conscience commune et sans rivale de tout le peuple.

«Le Sud a du beau bois » (4). Ce vers d'un poème ancien qui a su résumer tout l'aspect et la réserve de la terre chinoise du Sud devient chaque jour davantage un poème du passé. Jadis, lorsque ce poète écrivit ce vers qui allait se transmettre à travers les âges, il pensait, je crois, nommer un monde qui se perpétuerait jusquà la fin des temps. Le poète finit par mourir, et nous ne savons pas trop aujourd hui qui est l'auteur de ce bijou. Mais cela suffit que le Sud ait du beau bois, car cela suffit à ce que les gens, dans ce monde où la mort est indubitable. vivent comme toujours jusqu'au bout dans l'amour de la terre. Pourtant, le poète avait tort. S’il observait bien un monde à l'histoire vieille de cinq mille ans, face aux changements gigantesques du monde de ces cent dernières années, tout n'était juste qu'un commencement. Je partage l'opinion de $\mathrm{Gu}$ Hongmin: la Chine ancienne est restée cinq mille ans dans un état d'enfance.

\section{A propos des insectes et du monde nouveau}

Il y a peu, je suis allé résider un moment dans la campagne près de Kunming. A ma grande surprise jaa réalisé que les nuits n'y étaient plus remplies par le coassement des grenouilles. A la place s'étendait une terre au silence mortuaire absolument terrifiant. Les grenouilles avaient toutes été tuées par les insecticides. La belle terre du Sud où « reinettes peuplent mares et roseaux » est maintenant partout considérée comme une page blanche où «l'on peut peindre le plus heau et le plus neuf des tableaux ». Les villageois n'aspirent plus ardemment à l'automne, mais à la signature le lendemain d'un contrat de vente de leur terre à une compagnie de bâtiment. Un Sud qui n’a plus d'insectes peut-il encore s'appeler le Sud?

L'esprit esthétique et la perception du droit à l'existence libre des insectes vont à l'encontre de l'idéologie du profit en vogue dans la Chine moderne. Ainsi, il y a quelques jours, j’ai encore vu la télévision et les journaux appeler les gens à s'enrichir en attrapant les papillons de leur région. La loi morale (rendao) traditionnelle chinoise $n$ inclut pas les insectes, ni les animaux sauvages: "Une fois l'écurie brûlée, le Maître demanda : "Y a-t-il des blessés? Les chevaux ne comptent pas"». Et c'est ainsi que dans la nouvelle édition (1996) du Dictionnaire de chinois moderne. à mille-pattes est écrit « peut entrer 
dans la composition de médicaments». A grillon, «nuisible à l'agriculture ». A hanneton, "nuisible à l'agriculture ». A cerf, "la peau peut faire du cuir, les bois du jeune cerf peuvent entrer dans la composition de médicaments ». A criquet, « insecte nuisible». A cigale, «la cosse peut entrer dans la composition de médicaments $»$. A lombric, «leurs excréments peuvent enrichir le sol, animal utile ». C'est aussi pourquoi quand vous alle\% au zoo tous les animaux sont présentés au public en distinguant ceux que l'homme peut manger et ceux qui peuvent manger l'homme. C'est aussi pourquoi vous pourrez voir dans les jardins botaniques de nombreuses espèces catégorisées sur la base de leurs propriétés médicinales ou encore de leur qualité de matériaux de menuiseries. Le critère de tri pour les végétaux, les animaux sauvages et les insectes est leur utilité ou leur nuisance pour l'homme. siils peuvent être mangés ou šils peuvent servir à faire des médicaments. De telles valeurs déterminant les usages populaires sont aujourd’hui universellement répandues en Chine, et le résultat est qu'à force d'être mangés, les grands insectes et animaux sauvages ont quasiment disparu. Les gens en arrivent même maintenant à manger les fourmis et les vers de terre. Ceux qui étaient nuisibles ont déjà été tous mangés: manger ceux qui ne font aucun mal, c'est la loi morale. A cet égard, à quoi cela sert-il encore d'être triste? Si tu n'en manges pas, d'autres vont en manger, et pas seulement un ou deux. C'est dans les mours de le faire. Ne pas en manger ne sert donc à rien, «c"est la loi morale», et comme ça on a la conscience tranquille. Ce qui reste ce ne sont que des souvenirs. Et le souvenir, c"est juste une forme d'auto-sentimentalisme de l'imagination dans une existence de survie entourée par la mort. D'un autre côté, au moment même où l'on tue les insectes nuisibles de l'espèce des hannetons à coup d'insecticide. rien n’arrête les gens de lettres d'écrire des "Ah. lor jaune des cétoines dorées, les dieux de mes jeunes années" Ainsi. quand la Nature se remplit de morts, la langue chinoise est, elle, pleine de belles phrases qui chantent la Nature "éternelle ".

Le plus effrayant c"est que cette « loi morale» ne s"applique pas seulement aux insectes mais à l'ensemble du monde naturel et à toutes les choses anciennes. Que reste-t-il des "Quatre cents poèmes des Dynasties du Sud, mille terrasses dans les vapeurs et la pluie"? A nouveau lors d'un voyage de Kunming à Shanghai, jai vu ce que les industries du bâtiment étaient en train d'apporter, sans la moindre retenue, dans ce Sud éclatant de soleil: un monde standardisé de ciment et d'acier sans terrasses. Le

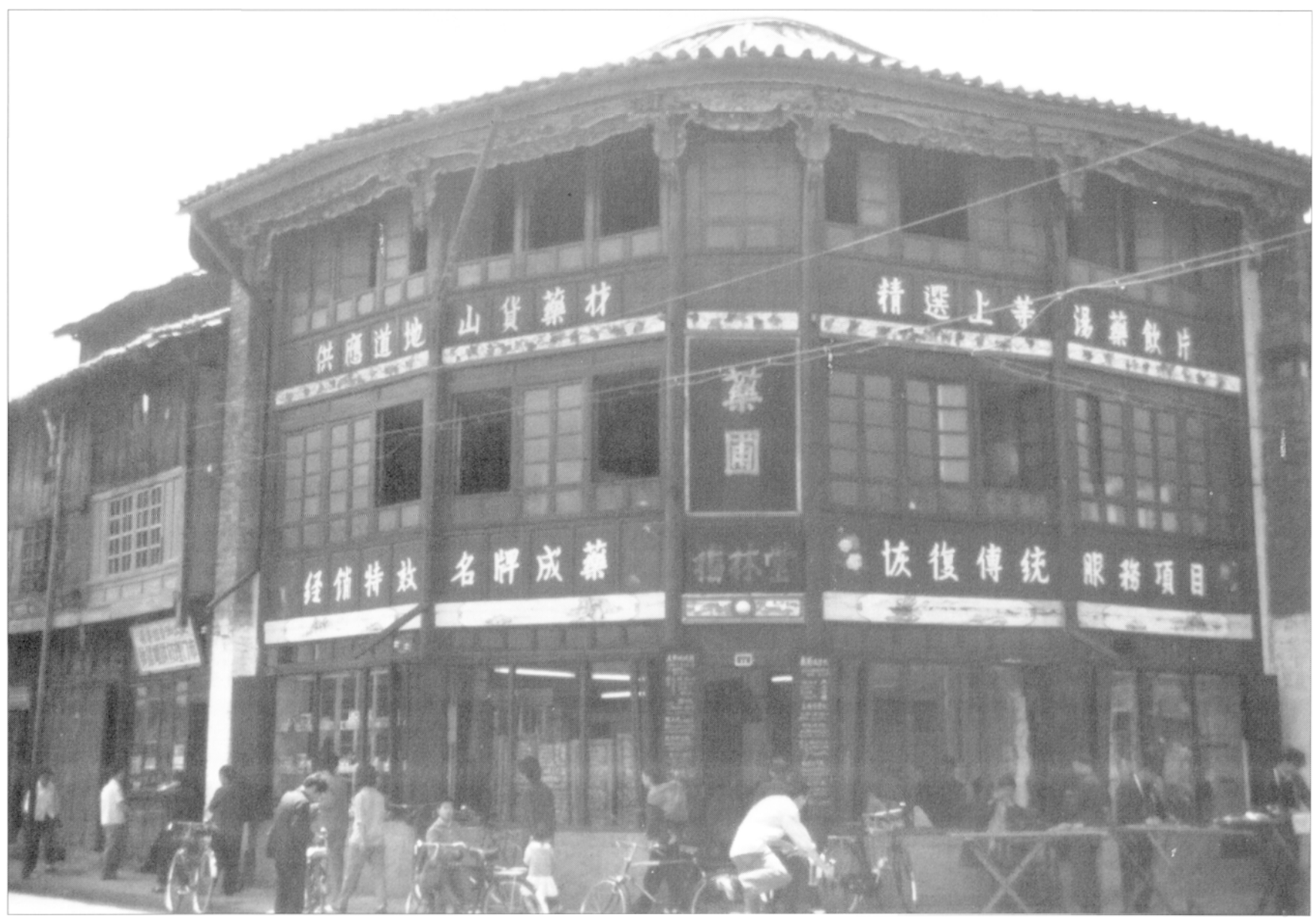

Les vieilles maisons de Kunming ont aujourd'hui presque toutes disparu 
Sud est en passe d'être recouvert par les carreaux de céramique. Toutes les villes, tous les quartiers résidentiels s'appliquent à faire la même chose. Les gens ne comprennent peut-être toujours pas leurs dialectes réciproques, mais la standardisation des marques extérieures de vie et d'architecture conduit déjà ces dialectes et les modes de pensée locaux à être considérés comme des obstacles à la modernisation. Le Sud pourra-t-il encore voir émerger des écrivains comme Li Jieren, Shen Congwen, Faulkner?

En Occident, le moderne est la création et la poursuite de la tradition, et c'est ainsi qu'en même temps que le moderne, on peut voir l'ancien monde volontairement préservé dans sa plus grande étendue. Le moderne témoigne ainsi d'une part de l'énergie créatrice de l'humanité, et atteste d'autre part de la grandeur des époques passées. Mais en Chine, les gens considèrent la tradition comme l'ennemi et l'obstacle à la modernité, comme une chose arriérée et réactionnaire qui entrave la vie des gens et dont il faut radicalement se distinguer. La révolution contre la tradition qui a duré tout ce siècle a atteint son extrême avec la "Révolution culturelle », et aujourd hui les Chinois se trouvent en rupture à tous les niveaux avec elle. La soi-disant page blanche où l'on peut peindre le plus beau et le plus nouveau des tableaux est déjà bien entrée dans le cœur des gens. La Chine traditionnelle, ce n'est déjà plus que les maisons à cour chancelantes qui s’apprêtent à être détruites, les vieilles murailles, les meubles anciens et les modes de vie des temps passés qui peuvent de moins en moins soutenir la comparaison. Le monde nouveau est quasi synonyme de destruction de la vie chinoise traditionnelle, et ses résultats de plus en plus évidents. Vous pouvez voir, par exemple, que dans toutes les villes de Chine. sans exception, on démolit les vieux quartiers. et que les vieux artisans et les vieilles boutiques qui y avaient

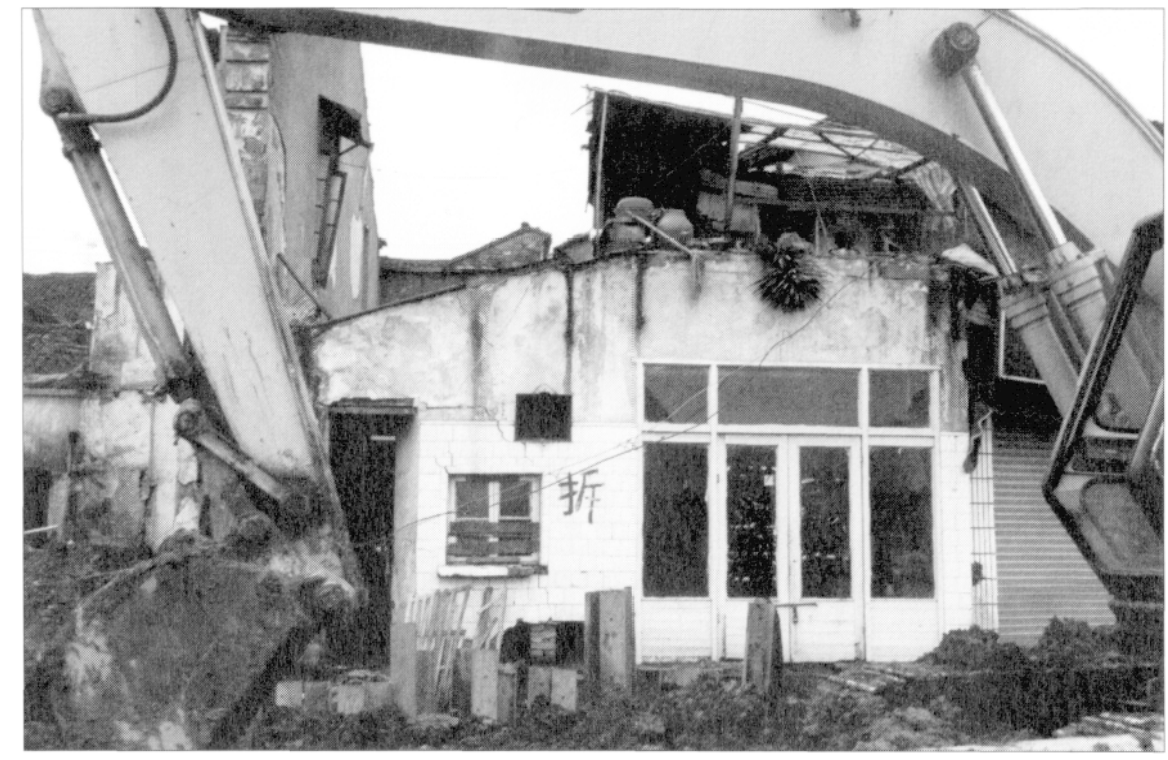

Pour construire un monde nouveau?

prospéré ont tous été anéantis. Dans dix ans, à part l'accent, le Nord et le Sud de la Chine n'auront plus aucune différence visible. Sans tradition à laquelle adhérer, sans poursuite de la tradition, qu'est-ce qui pourrait distinguer encore le Nord et le Sud du monde nouveau ? Faire passer la tradition quotidienne pour un monstre revient en fait à perdre toute norme générale. Et inévitablement, avec pour modèle une unique modernité, les gratte-ciels deviennent aux yeux de tous les symboles du monde nouveau. Mais quand la besogne aura été terminée, les gens se rendront compte un jour que la tradition, l'«ancien », c'est, à une époque moderne où tout est reproductible, l'unique fondement par lequel ils puissent conserver une spécificité, et que seules les âmes solitaires du passé, errant dans la désolation de l' «époque », leur permettent d'éviter l'insipidité d'une vie au goût de boîte de conserve.

Du moment où la culture radicale qui a fait suite au « Mouvement du 4 mai » atteint son point extrême, ses conséquences peuvent être catastrophiques. L'histoire d'avant 1976 a déjà témoigné de ce point. Quant à la suite. cela reste peut-être encore à prouver, mais les tenants et les aboutissants de la catastrophe sont déjà bien visibles. Aujourd hui, si la marche de la société chinoise vers le monde nouveau tourne à la radicalisation aveugle, le résultat en sera aussi, si ce n'est plus, catastrophique, car cela conduira non seulement à la perte des sources spirituelles des Chinois, mais aussi au bout du compte à la perte de leur propre terre. Va-t-on maintenant au nom du monde nouveau continuer la destruction radicale de la culture traditionnelle chinoise initiée par la Révolution culturelle? Je ne peux pas voir sans tristesse que l'on substitue toujours le nouveau à l'ancien. Déjà à l'époque de l'échec de Liang Sicheng, ce que l'on disait vouloir poursuivre n'était pas la destruction de la « vieille » culture mais la construction d'un monde nouveau. Le monde nouveau va, bien sûr, être bâti, mais ses bases reposeront sur la destruction totale du monde ancien. Ainsi, on rapportait dans le journal que l'ancienne demeure de Li Hongzhang à Hefei avait été démolie. La cause n'en est pas, bien sûr, un comprador qui vend le pays, ni la Révolution culturelle, mais encore les besoins du monde nouveau. On peut, cependant, se demander si les ténèbres d il y a vingt ans n'en sont pas aussi une des causes, car si cela avait été l'ancienne demeure de Lu Xun ou de Guo Moruo, aurait-elle eu la même fin?

Des journalistes écrivaient au jour 
de l'an qu'en se promenant dans ses grandes rues on se rendait compte que Kunming était devenue une nouvelle ville. Ils étaient ravis. En fait, Kunming ressemble aujourd'hui à un nouveau Tangshan après le tremblement de terre. En raison de son éloignement du centre culturel du pays, Kunming avait pu sauvegarder beaucoup de «vieux » des innombrables révolutions culturelles. Mais rien ne peut réfréner la soif de «neuf» des habitants. Tout le « vieux » visible qui avait survécu à la Révolution culturelle a maintenant été nettoyé. Pourtant Kunming est, dit-on, une " cité culturelle historique » réglementée au niveau national. C'est vraiment l'humour post-moderne du « $\mathrm{n}$ importe comment, ça marche». Ainsi, alors qu'on abattait une rue datant des époques Ming et Qing, on voyait bien quelques résidents mécontents, mais la cause de leur mécontentement, c'était de ne pouvoir emménager tout de suite dans des maisons neuves. Une fois que leur furent alloués des appartements avec un salon et quelques chambres, ils exultèrent et déménagèrent sur le champ. Il n'y avait absolument personne pour regretter ces portes et ces fenêtres ornées de toutes sortes d'animaux, d'oiseaux et d'insectes, auxquels les artisans avaient consacré tant de temps. Ou pour regretter les cours, les pavillons, les jardins chinois fantastiques évoqués dans les écrits de Zhu Duo, le "Nian nu jiao » (5) et les multiples sonnets de la poésie classique. Ainsi, un jour que je traversais cette rue vers midi, je l'ai vu remplie de poutres ornées et sculptées qui étaient utilisées par les ouvriers de la construction comme du bois d'cuure. Je me suis subitement souvenu du vieux poème de Sun Ranweng chantant les innombrables pêcheurs sur le fleuve (banjiang vu huo). Comme les choses ont changé ! Aujourd hui il ne reste rien de ces pêcheurs, et la "moitié de fleuve flamboyant de bateaux " est maintenant une mare d'eau sale et polluée. Je suis comme un vieil homme sentimental, $j a a i$ envie de pleurer.

La base philosophique du monde nouveau est le darwinisme, mais la fondation philosophique du monde chinois est l'union de l'homme et du ciel (tianren he yi). La relation que l'Occident explorateur du monde physique a avec la terre est de l'ordre du « découvreur», de «l'adversaire » et du « conquérant ». La valeur suprême y est que l'homme possède tout, réclame tout pour lui. Cela nécessite un Dieu qui puisse approuver et consoler l'arrachement de l'Occident à l'âme de la terre. Mais au XXe siècle, l'absence de Dieu a rendu tout cela problématique, c'est pourquoi Heidegger a lancé son appel à "l'établissement du sens poétique ». Or cet «établissement du sens poétique » est justement le mode d'existence de la Chine ancienne. La relation du monde chinois à la terre est l'affirmation que toutes les choses ont une âme et l'union de l'homme et du ciel. Le monde de l'esprit des Chinois n'est pas déposé auprès d'un Dieu, mais auprès de la terre. L'époque avance mais la terre reste toujours là où elle est. Si l'on continue à considérer aveuglément la terre comme une page blanche où l'on peut tracer le plus beau et le plus nouveau des dessins, cela conduira finalement à la brisure de l'union de l'homme et du ciel, à la fin de l'âme de toutes choses. La conquête de la terre a amené les Occidentaux à construire une église de la pénitence, mais une conquête identique ne conduira les Chinois qu'à perdre leur chez-soi et les racines de leur existence. Le Sud sans beau bois! Figurez-vous une nation qui a rempli la terre de poésie Tang et Song qui se retrouve sans lune à regarder parmi les pins, sans source claire coulant sur les pierres. Même si tout le monde vit dans des suites au Hilton, et que des berlines circulent sur toutes les routes, quel sens aura encore la vie!

Le monde nouveau veut rendre la vie des gens meilleure, mais une bonne vie doit être la libération du potentiel des gens à se créer une bonne vie. Aujourd'hui, le monde nouveau est compris comme le simple développement reproductif du bien-être matériel. C'est ainsi que dans ces régions de l'Asie marquées par le colonialisme et le mode de vie américains, il est juste une reproduction de ce qui se fait ailleurs. Or, justement, ce soi-disant monde nouveau contient une grande quantité de déchets de la modernisation. La créativité des gens y est devenue chose superflue. Mais s'il n'a pas de base culturelle (issue de la tradition, et pas seulement écrite), les gens ne pourront que devenir ses spectres en conserve. La perte aveugle de la raison qui le caractérise ne rendra pas la vie des gens meilleure, mais au contraire accélérera la mort de l'homme. La terre désolée décrite par Elliot, voilà ce à quoi le mouvement vers le monde nouveau amène l'Occident.

Le jour où les mots "ancien » et "lointain » disparaîtront, et que le monde sera devenu « récent ", «neuf » et sans plus d'animation ni de distance, alors les derniers jours du monde seront proches. Je le prédis.

1. Ma Yinchu. démographe chinois célèbre pour avoir le premier proposé un contrôle de la croissance de la population chinoise dans les années 1950. Ses suggestions furent rejetées au profit de la politique nataliste préconiséc par Mao arant d'être remises à l'honneur après l'arrivée au pouvoir de Deng Xiaoping.

2. Intellectuel, fils de Liang Qichao. qui défendit sans succès après 1949 un modèle de développement urbain de Pékin préservant les monuments et les portes des murailles de ville datant des Ming et des Qing.

3. Piece de Wu Han qui, à la suite d'une critique en règle de Yao Wenyuan, servit de cible pour le lancement de la Révolution culturelle.

4. Lexpression fait référence au théier.

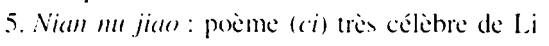
Qingzhao, poète (femme) de la dynastie Song. 